\title{
La rosa y la espina: expresiones musicales de solidaridad antiimperialista en Latinoamérica. El Primer Encuentro de la Canción Protesta en La Habana, Cuba, 1967*
}

\author{
The Rose and the Thorn: \\ Musical Expressions of Anti-imperialist \\ Solidarity in Latin America. The First Protest \\ Song Meeting in La Habana, Cuba, 1967
}

\author{
Juan Alberto Salazar Rebolledo** \\ (D) https://orcid.org/0000-0003-4166-9387 \\ Facultad de Filosofía y Letras \\ Universidad Nacional Autónoma de México, México. \\ j.s.rebolledo33@gmail.com
}

Resumen: El antiimperialismo en los años sesenta del siglo $\mathrm{xx}$ tuvo importantes componentes artísticos y culturales, la canción de protesta fue uno de

* Una primera versión de este trabajo fue presentada por el autor como ponencia en el Congreso Reinventing the Social: Movements and Narratives of Resistance, Dissension, and Reconciliation in the Americas de la International Association of Inter-American Studies, en la Universidade de Coimbra, Portugal, en marzo de 2018.

** Maestría en Historia, FFYL-UnAm. Líneas de investigación: historia cultural, historia intelectual.

Cómo citAR: Salazar Rebolledo, J. A. (2020). La rosa y la espina: expresiones musicales de solidaridad antiimperialista en Latinoamérica. El Primer Encuentro de la Canción Protesta en La Habana, Cuba, 1967. Secuencia (108), e1809. DoI: https://doi.org/10.18234/secuencia.v0i108.1809

\section{c) 98}

Esta obra está protegida bajo una Licencia Creative Commons Atribución-NoComercial 4.0 Internacional. 
ellos. Jóvenes de varios países latinoamericanos comenzaron a hacer música para denunciar, a la vez que confrontar, al imperialismo de Estados Unidos. Dichas expresiones musicales aglutinaban pronunciamientos de solidaridad y acompañamiento a luchas revolucionarias como la revolución cubana y hasta otras latitudes, como la guerra de Vietnam. Este artículo busca explicar lo anterior a partir de uno de los ejemplos más tempranos de este fenómeno en América Latina: el Primer Encuentro de la Canción Protesta en 1967 en La Habana, Cuba. Este evento marcó la pauta y desde entonces se configuraría como una manera casi programática de comprometerse políticamente desde la creación artística. Como demuestra este trabajo, la reunión en la isla fue un parteaguas para configurar la postura política de solidaridad en varios de los jóvenes músicos asistentes, como el caso del mexicano Óscar Chávez.

Palabras clave: antiimperialismo; solidaridad internacional; canción protesta; revolución cubana; Óscar Chávez.

Abstract: Anti-imperialism in the 1960s had significant artistic and cultural components, including protest songs. Young people from various Latin American countries began to make music to denounce, as well as confront, United States imperialism. These musical expressions brought together declarations of solidarity and the accompaniment of revolutionary struggles such as the Cuban Revolution and even those in other places such as the Vietnam War. This article aims to explain this through one of the earliest examples of this phenomenon in Latin America: the First Protest Song Meeting in 1967 in La Habana, Cuba. This event set the tone for what would become as an almost programmatic form of political engagement based on artistic creation. As this paper shows, the meeting on the island constituted a watershed in shaping the political position of solidarity in several of the young musicians who attended, such as the Mexican Óscar Chávez.

Keywords: anti-imperialism; international solidarity; protest songs; Cuban revolution; Óscar Chávez.

Recibido: 27 de enero de 2020 Aceptado: 3 de agosto de 2020 Publicado: 14 de diciembre de 2020 
E n América Latina, algunas expresiones musicales antiimperialistas de la segunda mitad del siglo xx tuvieron en común la búsqueda de "nuevos planteamientos sobre la tradición, lo popular y la revalorización de la identidad" (Velasco, 2007, p. 140). Este tipo de canciones tuvieron un especial auge en los años sesenta y setenta y muchos de sus creadores e intérpretes fueron jóvenes. Uno de los espacios internacionales más importantes de reunión e intercambio para estos músicos y compositores fue Cuba, en el marco de la guerra fría.

Como ha señalado el historiador Patrick Iber, la guerra fría tuvo una importante vertiente cultural en América Latina, a la que podríamos llamar guerra fría cultural (Iber, 2015). En este marco, la revolución cubana desempeñó un papel fundamental como un actor central en las dinámicas globales y regionales. Una de las manifestaciones de dicho proceso fue la temprana fundación de la Casa de las Américas el 28 de abril de 1959, a cargo de Haydée Santamaría. La principal misión de este organismo fue articular las dinámicas culturales y artísticas del continente. Varios proyectos fueron puestos en marcha para lograr dicho cometido, y el aspecto musical no fue la excepción, pues además se nutrió de los procesos en marcha en el resto de los países, como fue el rescate de la música popular, también llamado folclore, manifiesto en la Nueva Canción.

La intención de este artículo es presentar al Primer Encuentro de la Canción Protesta en La Habana, Cuba, en 1967, como un catalizador de las dinámicas musicales latinoamericanas de solidaridad con las luchas antiimperialistas de aquel momento, especialmente con la revolución cubana, la oposición a la guerra de Vietnam y la lucha por los derechos civiles en Estados Unidos. El Encuentro planteó un espacio de reunión para que varios músicos pudieran conocerse entre ellos e intercambiar canciones y visiones sobre su propio trabajo. La hipótesis central de este trabajo es que, para varios artistas, como el mexicano Óscar Chávez, este evento marcó un quiebre en su trabajo creativo, que en adelante estuvo caracterizado por el compromiso político y el acompañamiento a los movimientos sociales; por otro lado, promovió a Cuba y la Casa de las Américas como un núcleo de intercambio y producción fonográfica para la canción protesta latinoamericana y sus distintas derivaciones.

La confluencia de representantes de la canción popular en varios países latinoamericanos motivó a la Casa de las Américas para organizar el Primer Encuentro de la Canción Protesta, entre el 29 de julio y el 10 de agosto de 1967 en La Habana, Cuba. Además, el evento musical coincidió con la fundación de la Organización Latinoamericana de Solidaridad (OLAS) para buscar alter- 
nativas que hicieran frente al imperialismo estadunidense y el bloqueo económico que le había impuesto a la isla desde principios de la década: "experimentados profesionales y expertos de distintas partes del mundo, se juntaron para diseñar la manera de 'hacer olas' durante la Guerra Fría" (Fairley, 2013, p. 124), a la par del canto en contra del imperialismo.

Tanto en la organización del Encuentro como en la propia participación musical, los jóvenes latinoamericanos desempeñaron el papel principal. La idea era generar un correlato a la idea predominantemente mercantilista de la música en Estados Unidos. Tanto desde la música popular, que rescatara las tradiciones no incorporadas al mercado de la industria musical, como desde las composiciones claramente enfocadas en la denuncia de la explotación o la reivindicación de las luchas sociales.

Las canciones del Encuentro, al que asistieron 50 representantes de 18 países, cubrieron una amplia gama de temáticas: podían mostrar adherencia a algún movimiento social; recordar acontecimientos y personajes que hubieran hecho frente al imperialismo en algún momento de la historia de sus países; ensalzar algunas figuras como el "Che" Guevara y Ho Chi Minh; o reivindicar al "indio" latinoamericano como la abstracción del explotado potencialmente rebelde; así como la esperanza de un futuro mejor a través del combate a los explotadores por la vía revolucionaria.

Este tipo de reuniones internacionales de creadores tuvo como antecedente directo la proyección de Cuba como un núcleo orientador de la vida intelectual latinoamericana y sus circuitos de intercambio en los años sesenta, como lo ha estudiado la historiadora Claudia Gilman (2012). El "compromiso intelectual" establecido por escritores, artistas y algunos otros personajes relacionados con la cultura en la primera mitad de los años sesenta, sentó, en buena medida, las bases de la solidaridad artística subyacente a la canción protesta impulsada por la Casa de las Américas a partir de 1967.

Dentro del proyecto de la Casa se incluía una biblioteca, un premio literario anual, una editorial, una revista y un área dedicada a la investigación humanística y artística (Morejón Arnaiz, 2010, p. 67). La institución funcionó como un organismo validador de posturas políticas y culturales, inicialmente de los artistas y escritores cubanos. Conforme la Casa comenzó a adquirir el renombre del espacio eminente para la vida intelectual latinoamericana, a través de la gran diversidad de eventos que organizaba, también se convirtió en legitimadora del compromiso revolucionario de los intelectuales del resto del continente. Las páginas de la revista Casa de las Américas sirvieron para lo 
mismo, al abrir espacio para una gran diversidad de textos literarios, ensayísticos, políticos y reseñas de arte y cultura.

En ese horizonte, impulsadas por la Casa de las Américas resurgieron algunas tradiciones musicales cubanas de antaño, como la trova. Un grupo de jóvenes, entre los que se encontraban Silvio Rodríguez, Pablo Milanés, Noel Nicola, Sara González, y muchos otros, comenzaron a adquirir cierta fama a finales de los años sesenta, aunque fue posterior al Encuentro, pues no participaron en él. Para ellos, era "a través de una profunda revalorización de nuestras tradiciones, de nuestro acercamiento desprejuiciado y crítico a los valores más genuinos de nuestro acervo cultural, como podremos desarrollar un arte verdaderamente revolucionario" (Nicola, 1975, pp. 10-12).

La "vieja trova" incluía una amplia gama de formas musicales como el bolero, la guaracha, la guajira, el bambuco y el son. Su característica principal era la sencillez de su musicalización con una sola guitarra. "La 'nueva trova' [también] abrazó una amplia variedad de estructuras tradicionales, temas y estilos, pero los compositores jóvenes incorporaron [además] algunos ritmos contemporáneos internacionales en su música” (Benmayor, 1981, pp. 12-13).

En general, el tránsito de los jóvenes músicos latinoamericanos comenzó con la revitalización de "los vestigios de repertorios y poemas heredados de largas tradiciones sonoras y literarias”. Este proceso implicó, según Tejeda (2017), "Empaparse de una cultura, beber de saberes tradicionales, bañarse de ciertos sonidos, mojarse en las aguas populares, nutrirse de imágenes, alimentarse de determinados procedimientos, reflejar ciertos resplandores, conservar peculiares colores de pueblo, narrar historias inconclusas, tejer hilos aún sueltos, fluir, confluir e influir en espacios previamente habitados con seres preexistentes" (p. 76).

Tanto estas manifestaciones artísticas como la Casa de las Américas que las promovía eran eminentes expresiones del aspecto cultural de la guerra fría. La creación de dicha institución podría haberse inspirado en la disputa que había comenzado a finales de los años cuarenta entre el Congreso por la Libertad de la Cultura - respaldado por Estados Unidos- y el Consejo Mundial por la Paz -promovido por el bloque soviético-, que colocaron las discusiones al respecto de las premisas para la creación literaria al centro del debate a lo largo de la segunda mitad del siglo xx. Mientras tanto, en América Latina varios regímenes autoritarios y represivos se instalaron a partir de los años cincuenta a través de golpes de Estado, dictaduras y Juntas militares. En este contexto se situaba el entusiasmo intelectual latinoamericano por una 
institución de inspiración revolucionaria como la Casa de las Américas, que aparentemente ofrecía las libertades y condiciones negadas en buena parte del resto del continente. ${ }^{1}$

Varios músicos latinoamericanos habían comenzado tempranamente su vinculación con la revolución cubana a través de la Casa de las Américas. Sin embargo, no había una integración regional en este aspecto como sí en el intelectual, por lo que el Encuentro de la Canción Protesta de 1967 buscó generar dichas dinámicas para ampliar los alcances de la red de solidaridad con las luchas antiimperialistas en el plano de la guerra fría cultural latinoamericana. Para precisar la importancia de las expresiones musicales en este ámbito, a continuación haremos un breve recuento de algunas posturas sobre las implicaciones de la canción protesta y su vinculación con los movimientos sociales.

\section{LA CANCIÓN PROTESTA COMO EXPRESIÓN DE SOLIDARIDAD ANTIIMPERIALISTA}

Quizá una de las más tempranas reflexiones en el siglo xx sobre las implicaciones de la creación artística musical enmarcada en contextos sociales haya sido la del estadunidense Elie Siegmeister en Música y sociedad de 1938. En su texto, Siegmeister criticaba el "concepto de la música como puro entretenimiento" (Siegmeister, 1999, p. 29) para enfatizar la amplitud de las funciones sociales que la música había tenido a lo largo de la historia. Por ejemplo, en el caso de la Edad Media, destacaba las "canciones anticlericales [que] reflejaban el resentimiento creciente de la gente de todas las clases durante los siglos XII y XIV contra la codicia, la corrupción y los abusos escandalosos de la Iglesia” (p. 48).

Para Siegmeister, la música fue cumpliendo diversas "funciones" conforme a las necesidades de las clases sociales que la instrumentaron como

${ }^{1}$ La Casa de las Américas definitivamente no puso fin a las disputas culturales de la guerra fría. Sin embargo, sí reubicó la discusión en el plano regional, pues la revista Mundo Nuevo - auspiciada por Estados Unidos y en cierta medida derivada del Congreso por la Libertad de la Cultura- comenzó a rivalizar directamente con los postulados del compromiso revolucionario cubano desde su primer número de julio de 1966.

Para una revisión detallada de las polémicas entre las revistas Casa de las Américas y Mundo Nuevo, véase Morejón Arnaiz (2010). Para un panorama más extenso sobre la guerra fría y, en específico su vertiente cultural, véanse Iber (2015); Rojas (2018); Pettinà (2018). 
herramienta de denuncia, para llamar a la unión en las luchas sociales, o bien, en el momento en el que escribía, para "unificar el aprendizaje y la popularidad en un arte que debe convertirse en un instrumento amplio de ilustración y cambios sociales", cuya principal tarea sería la de enfocar e intensificar el "sentimiento de solidaridad entre las grandes masas populares" (p. 101).

El sentimiento de solidaridad anotado por Siegmeister fue sin duda el componente central del Encuentro de la Canción Protesta de 1967, sobre todo a partir de la enunciación de los principios orientadores del antiimperialismo en la denuncia de la guerra de Vietnam, el racismo en Estados Unidos, o la propuesta de organismos como Olas para defender a la revolución cubana. Cada una de estas consignas revestía la necesidad de expresar artísticamente la solidaridad de los asistentes al Encuentro desde sus producciones musicales; aun cuando es claro que cada una de las luchas tenía sus particularidades y diferencias, este era el "sentimiento" aglutinante que sustentó y promovió el evento.

A la par de las particularidades de las luchas aludidas hubo también una serie de características en común entre las expresiones artísticas, que Paulo de Carvalho-Neto llamó "el folklore de las luchas sociales" (Carvalho-Neto, 1973), y distinguió las siguientes posibilidades: ataque y defensa u opresión y resistencia. Entre las manifestaciones culturales que contemplan estas posibilidades estaba el "folklore de protesta", caracterizado por John Greenway, a partir de la tradición estadunidense, como una manifestación de "amargura y odio contra el opresor, y su firme decisión en fortalecer dichos sentimientos y en batallar por una vida mejor" (Carvalho-Neto, 1973, p. 24).

Si acudimos a la tradición estadunidense de la canción protesta, podemos ubicar las primeras manifestaciones de este tipo de música como parte de los movimientos obreros y sindicalistas desde finales del siglo xIx. En aquellos casos, la canción protesta era un acompañamiento de las sesiones de discusión y su función era principalmente didáctica, sobre los problemas que estaba confrontando la organización o en un sentido más o menos propagandístico como una invitación a la militancia o a apuntalar las huelgas. Aun cuando estos elementos no se separaron por completo de los fines y propósitos de la canción protesta en su dimensión de solidaridad con movimientos como el antiimperialismo, para Dorian Lynskey el gran hito de este tipo de expresiones musicales fue "Strange Fruit" de Billie Holiday en 1939, que "no fue la primera canción protesta, pero sí fue la primera que trasladó un mensaje político explícito al mundo del espectáculo" (Lynskey, 2011, p. 25). Otros representantes de la canción protesta en Estados Unidos, a medio camino entre 
la militancia y el desarrollo de un nicho del espectáculo musical en el siglo xx fueron: Woody Guthrie, Peete Seeger, Phil Ochs, Bob Dylan, Joan Báez, entre varios más, aun cuando no fueron pocos quienes en distintos momentos se desmarcaron del término "canción protesta" por considerarlo un encasillamiento limitado.

En este punto de la diferenciación entre la canción protesta espectacularizada y la operativa para la militancia, cabe retomar el señalamiento de Édison Carneiro sobre "cuán ineficiente es en la práctica" el folclore o la canción de protesta, pues se trata solamente de "un arma rudimentaria, tan rudimentaria que puede ser tolerado por el aparato de represión del Estado", que de Carvalho-Neto complementó diciendo que "no se hace una revolución únicamente con el folklore de protesta" (Carvalho-Neto, 1973, p. 30). Si consideramos esta categórica proposición, cabe preguntarnos cuál es entonces el papel de la canción protesta en su relación con los movimientos sociales. Para lo cual, Paul Almeida ofrece una consideración en torno al proceso de "framing", que podemos entender como una manera de configurar los marcos y referentes de una movilización u organización, en la que la canción protesta tiene una serie de relevantes potencialidades en el plano ideológico, cultural, simbólico y normativo (Almeida, 2019, pp. 81-82).

Paul Almeida apunta tres posibilidades para la canción protesta al "enmarcar" las características del movimiento social del que forman parte: el diagnóstico, el pronóstico y la motivación. Es decir, lo primero es señalar las causas que hacen necesaria la movilización, después es necesario apuntar las potencialidades que se abren al manifestarse y, finalmente, impulsar a quienes escuchan para que se unan al movimiento y a la lucha. Los mecanismos para hacerlo implican cuatro fases (Almeida, 2019, pp. 84-87): conectar y poner a interactuar sentimientos de opresión inconexos (difusión del diagnóstico desde el plano artístico y cultural); amplificar el marco de referencias por medio del énfasis en dichas conexiones (emparentar sentimientos y valores culturales entre grupos diversos); promover la unión y coalición entre movimientos al extender el marco; reorientar o corregir aspectos del marco de referencia del movimiento para darle mayor solidez y conexión con sus integrantes.

El proceso señalado por Almeida puede ser observado en distintos momentos del Encuentro de la Canción Protesta de 1967, pero también hay particularidades en el ámbito latinoamericano que quedan fuera de estas consideraciones. Una de ellas es la anotada por José Castillo Farreras respecto de que, si bien la conexión con los movimientos sociales en ocasiones es opera- 
tiva directamente con la enunciación literal de agravios, acciones o principios aglutinantes, también es cierto que "la protesta no sólo se manifiesta a través de la denuncia expresa. La simple mención de un hecho cultural tradicional puede ser una forma de protesta" (Carvalho-Neto, 1973, pp. 40-41). En ese sentido, el rescate de géneros musicales, instrumentos, ritmos, atuendos o la mirada a aspectos olvidados de la "tradición", eran ya expresiones de la canción protesta latinoamericana, como en el caso del Movimiento del Nuevo Cancionero en Argentina, cuyo manifiesto de 1963 señalaba: "la necesidad de tomar la 'palabra del pueblo', de un pueblo en un tiempo contemporáneo, no en un pasado idealizado como se muestra en un folclor tradicionalista. Entienden la tradición como un elemento dinámico, no estático ni esencialista, reivindicando el hombre común y sus problemáticas sociales" (García y Greco, 2017, p. 26), así como la diversidad local y regional de cada una de las expresiones "tradicionales", opacadas por la homogeneización nacionalista y, desde luego, el influjo del imperialismo cultural de Estados Unidos.

Así como en Argentina, en otros países de América Latina había comenzado en los años sesenta un proceso de revalorización de la música popular de cada lugar. Si bien algunos casos, como el argentino, tenían un proceso más avanzado -estructura y orientación política- que otros, el Primer Encuentro de la Canción Protesta de 1967 leyó bien la necesidad de impulsar estas inquietudes locales para vincularlas, en primera instancia, regionalmente, pero también con músicos de otras latitudes inmersos en un horizonte similar de tránsito del folclore a la protesta.

\section{EL PRIMER ENCUENTRO DE LA CANCIÓN PROTESTA EN LA HABANA, CUBA, EN 1967}

El Primer Encuentro de la Canción Protesta se llevó a cabo entre el 29 de julio y el 10 de agosto de 1967 en La Habana, Cuba, con 50 representantes de 18 países. La vocación política del evento musical estuvo claramente orientada a manifestar expresiones de solidaridad con distintos movimientos sociales, como la revolución cubana, el movimiento por los derechos civiles en Estados Unidos, y la oposición a la guerra de Vietnam. Sin embargo, cada uno de los asistentes manifestaba particularidades, por ejemplo, en el aspecto formal de sus canciones, inspiradas o retomadas de la música popular de sus sitios de procedencia, o en el nivel de compromiso político que estaban dispuestos a 
expresar en sus obras artísticas. Comenzaremos por hablar de algunos de los procesos locales de esta vertiente musical para contextualizar el papel de cada músico en el Encuentro, lo cual nos permitirá abordar también la postura colectiva propuesta por los asistentes en las discusiones en torno a las prerrogativas de la canción protesta.

Antes de enfocar el lente en América Latina, cabe destacar que entre los asistentes al Encuentro hubo una gran cantidad de europeos. En primera instancia, esto hablaba de un interés vinculatorio aún más ambicioso por parte de la esfera cultural de la revolución cubana, que buscaba convertir a la isla en el núcleo orientador de la expresión política musical de la canción protesta en el mundo. A la vez, esto permite revalorar a cabalidad la guerra fría cultural latinoamericana como un proceso con características locales específicas, no necesariamente dependientes ni determinadas por la bipolaridad de las potencias.

Algunos de los europeos asistentes al Encuentro fueron: de Italia, Iván Della Mea, Leoncarlo Settimelli, Giovana Marini, Elena Morandi y Meri Franco Leo, quienes combinaban un repertorio de baladas tradicionales italianas, canciones antifascistas y de asuntos políticos contemporáneos; de la península Ibérica, el portugués Luis Cilia y el valenciano Raimón, a quien llamaban "el poeta de izquierda por excelencia" (Microbiografías y opiniones, 1968, p. 39), y que se mantenía activamente vinculado con estudiantes, sindicatos y centros obreros, como miembro del movimiento musical de finales de los años cincuenta, la "Nova Cançó" Catalana; de la República Democrática Alemana, Gerry Wolf; de Francia, Claude Vinci; y de Gran Bretaña, Ewan Mc Coll y Peggy Seeger, así como el grupo Los Críticos, integrado por la maestra Sandra Kerr, el químico Terry Yamell y el electricista John Faulkner, quien reivindicó del Encuentro el valor de "encontrarnos con gente de Latinoamérica, ya que es ésta para nosotros un área bastante desconocida" (Microbiografías y opiniones, 1968, p. 39).

A pesar de lo evidente que podría resultar el desconocimiento europeo de la canción protesta latinoamericana, este tipo de afirmaciones no fueron menos frecuentes entre los músicos de América Latina. Un ejemplo fue el del uruguayo Daniel Viglietti, quien planteaba en los siguientes términos la desconexión: "Vivíamos aislados. Nos éramos desconocidos, aunque nuestras voces, desde hace tiempo moldeaban un mismo rostro de lucha y rebeldía". (Viglietti, 1968, p. 15). 
Daniel Viglietti, Quitín Cabrera, Aníbal Sampayo, Yamandu Palacios, Marcos Velázquez, Los Olimareños, Carlos Molina y Alfredo Zitarrosa integraban el contingente nacional más extenso, el uruguayo. Algunos de los elementos en común entre ellos fueron su confluencia con los géneros de la música tradicional campesina del Río de la Plata, reelaboraciones locales de manifestaciones hispanas y luso-brasileñas -milonga, zamba, chamarrita, entre otros- $y$ "un fuerte vínculo con las figuras emergentes en la Argentina, las integrantes del llamado "Nuevo Cancionero" y la "Nova Cançó" Catalana (Fornaro Bordolli y Malán, 2017, pp. 44-45). Viglietti, por ejemplo, combinaba su destreza en la guitarra clásica con adaptaciones de poemas de Federico García Lorca, Rafael Alberti, César Vallejo, Nicolás Guillén, Amanda Berenguer, Washington Benavides, entre otros. Además, en el encuentro cantó una de sus composiciones más populares, "Canción para mi América", que apelaba a la unidad antiimperialista latinoamericana y la combatividad de la música que debería acompañarla (Viglietti, 1969):

\author{
América está esperando \\ Y el siglo se vuelve azul \\ Pampas, ríos y montañas \\ Liberan su propia luz \\ La copla no tiene dueños \\ Patrones no más mandar \\ La guitarra americana \\ Peleando aprendió a cantar
}

Otro de los uruguayos, Aníbal Sampayo, trabajó en la Radio Paysandú como operador y locutor, desde donde difundió la música tradicional paraguaya, argentina y uruguaya. Su participación en los medios de comunicación le permitió vincularse con los movimientos musicales de otros países, como, por ejemplo, cuando participó en 1961 en la fundación del Festival Nacional de Folklore de Cosquín en Córdoba, uno de los más importantes eventos musicales de esta corriente en Argentina. Más tarde, en 1972, tras un salto de la canción protesta a la militancia directa en los movimientos revolucionarios, fue arrestado y encarcelado por ser miembro del Movimiento de Liberación Nacional, Tupamaros. 
Entre los argentinos estaba Ramón Ayala, quien ya tenía cierta fama continental desde principios de los años sesenta, cuando pertenecía al trío Sánchez, Monges y Ayala. En 1962 comenzó una gira solista por varias ciudades de Europa y de Cuba, lo cual le permitió vincularse desde entonces con las instancias culturales de la isla. Una de sus canciones más populares, "El mensú", hablaba de la vida de un campesino (un "mensú") con alusiones alegóricas a la explotación y la posibilidad de superarlas colectivamente (Ayala, 2013):

$$
\begin{aligned}
& \text { Río, viejo río que bajando vas, } \\
& \text { quiero ir contigo en busca de hermandad, } \\
& \text { paz para mi tierra cada día más, } \\
& \text { roja con la sangre del pobre mensú. }
\end{aligned}
$$

Óscar Matus, Celia Birembaum y Rodolfo Mederos, también asistentes al Encuentro, eran miembros y fundadores del "Nuevo Cancionero" argentino, al que ya hemos aludido previamente y que se planteó como una postura crítica frente al llamado "boom folklórico", principalmente a raíz de la primera edición del Festival Nacional de Folklore de Cosquín que se realizó entre el 21 y el 29 de enero de 1961. Varios cantantes de música popular, como los argentinos Santos Alcides Sarmiento, Jaime Dávalos y el uruguayo Aníbal Sampayo, convocaron al evento. A lo largo de la década, también se fueron sumando otros artistas más jóvenes, como Horacio Guarany, Alfredo Zitarrosa, Los Chalchaleros o Jorge Cafrune, quien popularizó la icónica composición de Luis Profili, "Zamba de mi esperanza”, que tenía como base la estructura clásica de la zamba (Cafrune, 1964):

$$
\begin{aligned}
& \text { Hundido, en horizonte } \\
& \text { soy polvareda que al viento va } \\
& \text { Zamba, ya no me dejes } \\
& \text { yo sin tu canto no vivo más } \\
& \text { Zamba, ya no me dejes } \\
& \text { yo sin tu canto no vivo más }
\end{aligned}
$$

En esta composición, Jorge Cafrune no sólo planteaba un rescate formal de los ritmos propios de una región de su país natal, sino que en la propia composición lírica expresaba el profundo, y hasta vital, significado que la zamba adquiría para un joven de la provincia argentina de Jujuy. El contacto 
con las diversas regiones y tradiciones musicales locales fue también uno de los componentes centrales del "Nuevo Cancionero".

Óscar Matus, uno de los fundadores del "Nuevo Cancionero", había comenzado a hacer giras por Argentina desde finales de los años cincuenta. En 1957, de paso por Tucumán, conoció a la entonces joven Mercedes Sosa con quien contrajo matrimonio. Sosa describía así el impacto de la llegada de Matus a Tucumán: "El folklore era una cosa de paisajitos; con las canciones de Matus y de Tejada Gómez se descubrió que importa el paisaje, pero mucho más ha de importar el hombre [...] Y así cambió el folklore con la llegada de Matus, cambió la Marta [Mercedes Sosa] y su repertorio" (García y Greco, 2017, p. 26). Un poco más tarde, entre 1964 y 1967, gracias a sus extensos vínculos con los músicos sudamericanos, Matus también pudo establecer su fugaz sello discográfico Producciones Matus, con cierto apoyo del Partido Comunista Argentino. Esta productora permitió dar a conocer a varios artistas que grabaron ahí sus primeros álbumes, lo cual llamó la atención de las autoridades cubanas para invitar a Matus al Encuentro de 1967 (García y Greco, 2017, pp. 32-37). El capital de Matus, con sus conexiones previas a través de la disquera, podía ayudar a tejer redes más sólidas entre los músicos latinoamericanos desde la Casa de las Américas.

Un caso que planteaba un atractivo similar al de Matus para las autoridades cubanas del Encuentro fue el de los chilenos Ángel e Isabel Parra, hijos de Violeta Parra -quien se había suicidado a principios de 1967-, uno de los grandes iconos de la música popular chilena, junto con Víctor Jara, Patricio Manss y Rolando Alarcón, caracterizada por "sus líricas poéticas, el uso de instrumentos indígenas latinoamericanos y un mensaje político de justicia social y antiimperialismo" (Spener, 2016, p. 140).

Ángel e Isabel fundaron en 1965 La Peña de los Parra, un "local destartalado [que] era un lugar de paso para músicos, escritores, estudiantes políticos e intelectuales que deseaban escuchar folk en su propio ambiente informal y bullicioso" (Lynskey, 2011, p. 339). Tras la asistencia al Encuentro, Ángel e Isabel Parra adquirieron aún más contactos e infraestructura que finalmente les permitió fundar su propio sello discográfico en 1968, una plataforma fundamental para la difusión de esta vertiente musical en Sudamérica. A la par, el productor musical Camilo Fernández acogió la "nueva canción" chilena en su disquera independiente Arena/Demon, también a mediados de la década. En 1968, la Discoteca del Cantar Popular (DicAP) le grabó el disco Por Vietnam al grupo Quilapayún para presentarlo en el Festival Europeo de 
la Juventud; tanto ellos como Inti-Illimani alcanzaron gran difusión en América Latina y Europa.

Es importante destacar que la tradición musical chilena se remontaba a eventos musicales ligados con los ciclos agrícolas y de cosecha, sin una consigna política tan clara como la del Encuentro de 1967. Algunas de las reuniones musicales en torno a la producción eran: "[las] de la sandía (Paine), la espiga (Yungay), la guinda (Romeral), la vendimia (Molina) y las bayas (Río Negro)" (González, 2017, p. 5), por lo que, para los jóvenes músicos, las alusiones al rescate de las tradiciones del "pueblo chileno" eran una manera incluso telúrica de resistir frente al avasallador imperialismo cultural.

El gran impulso al rescate del folclor chileno se remonta al menos a mediados de los años cincuenta. Los festivales organizados en 1956 y 1957 por el Sindicato de Guitarristas en el Teatro Caupolicán de Santiago, dieron cuenta de ello. En aquellos participaron grupos como el Conjunto Fiesta Linda, Raúl Gardy, Los Hermanos Lagos, Segundo Zamora y Guadalupe del Carmen, entre otros. Estos eventos y estos grupos sentaron un fecundo precedente para el interés de los jóvenes del país sudamericano en la siguiente década.

En el transcurso de los años sesenta, la vertiente juvenil de esta corriente musical se hizo más evidente con los festivales estudiantiles de la canción chilena, como el Festival de la Guinda en Romeral (1966), el Festival Folclórico de la Patagonia (1968), el Festival Nacional de Folclor de Río Claro (1970), el Festival del Huaso de Olmué (1970) y el Festival Nacional de Folclor de San Bernardo (1971), surgidos a partir del abandono del folclor por parte del Festival de Viña del Mar, organizado desde 1960. La composición de Violeta Parra, Que vivan los estudiantes, que se hizo famosa por su hijo Ángel e interpretada estelarmente en el Encuentro de 1967 en La Habana, planteaba la vinculación entre la canción protesta chilena y los jóvenes estudiantes (Parra, 1965):

\footnotetext{
Que vivan los estudiantes, jardín de las alegrías.

Son aves que no se asustan de animal ni policía, y no le asustan las balas ni el ladrar de la jauría. Caramba y zamba la cosa, que viva la astronomía.
} 
Víctor Jara, amigo cercano de Violeta Parra desde finales de los cincuenta y uno de los grandes impulsores de la música popular chilena, planteó con claridad las intenciones antiimperialistas de las expresiones musicales que impulsaban, dirigidas con particular atención a los jóvenes latinoamericanos, en busca de despertar su rebeldía y la conciencia de su situación y del mundo que les rodeaba (Vilches, 2004): "Claro, nosotros cantamos la verdad, y la industria del disco y la penetración cultural del imperialismo norteamericano en todo nuestro continente tratan de darle con la canción una especie de evasión, de conformismo y enajenación a la juventud, para que no se dé cuenta dónde está y dónde vive, cuál es su papel real, para adormecer la rebeldía natural en el joven" (p. 204).

En 1959, Jara visitó Cuba como parte de una gira teatral y tuvo una audiencia con el "Che" Guevara, por lo que suponemos había también una conexión cercana con la isla (Lynskey, 2011, p. 338). Más tarde, en 1964, Jara se vinculó estrechamente con el presidente del Partido Socialista Chileno, Salvador Allende, y participó musicalmente en su campaña presidencial de aquel año, lo cual nos permite enfatizar el vínculo entre la canción protesta y los movimientos políticos y sociales latinoamericanos. Quizá las actividades al interior del partido no le permitieron a Jara asistir al Encuentro en Cuba, o su participación en las redes ya estaban en un nivel distinto al del resto de los asistentes. Como haya sido, no hay rastros claros de que se le hubiera extendido la invitación.

Finalmente, la lista de quienes sí asistieron al Encuentro se completaba con Van Loc, Tran Dung y Phan Doung, de Vietnam del Sur; Julius Lester, Barbara Dane e Irwin Silber, de Estados Unidos; Jean Lewis, de Australia; Onema D'Jamba Pascal, del Congo Kinshasa; Martha Jean Claude, de Haiti; Carlos Puebla, de Cuba; Los Guaraníes, de Paraguay; Nicómedes Santa Cruz, de Perú; Rolando Alarcón, de Chile; y, por último, los mexicanos José González Márquez y Óscar Chávez, en cuya experiencia y contexto ahondaré en la siguiente sección de este trabajo.

El poster del Encuentro, "La rosa y la espina", fue diseñado por el joven cubano Alfredo Rostgaard. En él se manifestaba artística y poéticamente, por un lado, la vertiente romántica de esta corriente musical, y, por otro, su aspecto combativo, doliente y de confrontación. Ángel e Isabel Parra retomaron la imagen para convertirla en el icono del sello musical de la Peña de los Parra, al que ya hemos aludido, y perpetuaron la prolongada asociación del símbolo con la canción comprometida. 
El Encuentro tuvo al menos dos componentes: el aspecto propiamente de las presentaciones musicales, y la discusión artístico-política sobre el papel social de la "canción protesta", término que pretendía aglutinar la diversidad de manifestaciones musicales presentadas, pero que dividió posturas, pues muchos de los artistas no se sentían del todo identificados con él.

Algunas de las líneas principales de la resolución final del Encuentro fueron: considerar la búsqueda de la calidad artística como una actitud revolucionaria; apoyar la lucha proletaria y estudiantil contra la explotación en los países capitalistas "fiel aliada del imperialismo", así como "la creciente militante lucha del pueblo negro de los Estados Unidos contra todas las formas de discriminación y explotación"; defender "a la Revolución Cubana, que ha señalado el verdadero camino que deben tomar los pueblos de Asia, África y América Latina para liberarse", es decir, tomarla como guía y referente para la vía revolucionaria del Tercer Mundo; $y$, finalmente, "demandar y exigir un inmediato e incondicional cese de los bombardeos a Viet Nam del Norte, la retirada total de todas las fuerzas de los Estados Unidos de Viet Nam del Sur [sic]", ya que el rechazo a la guerra de Vietnam fue uno de los principales motivos para convocar a la reunión (Resolución Final del Encuentro de la Canción Protesta, 1967).

Pero quizá la mayor consigna fue la de la definición básica de su labor como "trabajadores de la Canción de Protesta":

Los trabajadores de la Canción de Protesta deben tener conciencia de que la canción, por su particular naturaleza, posee un enorme poder de comunicación con las masas en tanto que rompe las barreras que, como el analfabetismo, dificultan el diálogo del artista con el pueblo del cual forman parte. En consecuencia, la canción debe ser un arma al servicio de los pueblos y no un producto de consumo utilizado por el capitalismo para enajenarlos (Resolución Final del Encuentro de la Canción Protesta, 1967).

Este planteamiento fue el que permitió tender puentes más sólidos entre el rescate de la música popular y la vertiente directamente de protesta. La confrontación con la música mercantilizada desde el rescate de la música popular fungió como una de las consignas centrales. En cierta medida, esta característica fue aglutinante de las expresiones musicales, que adquirían así su carácter antiimperialista, al estar fuera del circuito industrial de la música y en contra de la cultura de consumo promovida por el imperialismo cultural. 
En varios momentos, los participantes en el Encuentro coincidieron con las reuniones de la Olas, y quizá de estos contactos surgió la impronta de formar también una organización de defensa de la canción latinoamericana: el Centro de la Canción de Protesta, para "crear una especie de "Archivo General del Folklore", "distribuir todo el material que puedan recolectar" y para "la grabación de dos discos de larga duración (longplaying) que incluyeran a los participantes del Encuentro de la Canción de Protesta 1967" (Última asamblea de la canción protesta, 1967). Uno de ellos fue Óscar Chávez, junto con José González Márquez, los únicos mexicanos que asistieron al Primer Encuentro de la Canción Protesta.

\section{ÓSCAR CHÁVEZ: LA EXPERIENCIA DE UN JOVEN MÚSICO ANTIIMPERIALISTA EN CUBA}

Desde principios de los años sesenta, Óscar Chávez conjugó su trabajo en el teatro experimental con el rescate de la canción tradicional mexicana. Una de las fuentes para abrevar de esta corriente fue su familia. Su padre guitarrista le enseñó muchas piezas musicales que incorporó al repertorio que interpretaba en escuelas de la Universidad Nacional Autónoma de México (UNAM) y el Instituto Politécnico Nacional (IPN), con su amigo José González Márquez. "Y dentro de la canción tradicional hay mucha canción de índole política. Canciones muy bien hechas, muy acertadas, con una gran carga de ironía y de humor". ${ }^{2}$

En 1963, Chávez en la voz y González Márquez en la guitarra, grabaron su primer disco con Polydor, Herencia Lírica Mexicana. La mayoría de las piezas eran corridos, sones, coplas, canciones y romances trovadores, que hablaban del amor, el despecho o bucólicas costumbres de la provincia mexicana, como El lirio, una composición romántica (Chávez, 1963):

Hay un lirio que el tiempo lo consume $Y$ hay una fuente que lo hace enverdecer

Tú eres el lirio y dame tu perfume Yo soy la fuente y déjame correr

${ }^{2}$ Entrevista al cantante y compositor Óscar Chávez, realizada por Juan Alberto Salazar Rebolledo, 26 de febrero de 2018. Ciudad de México, México. 
El disco era presentado así en la contraportada: "no se pretende que este volumen sea considerado como folklore, sino como una interpretación del mismo destinada a revivir en la memoria y el gusto del México actual los valores de su prodigiosa tradición musical" (Chávez, 1963), un planteamiento nada alejado de los lineamientos propuestos por el manifiesto del Nuevo Cancionero argentino en el mismo 1963.

Los micrófonos radiofónicos de Radio Universidad también contaron con la participación de Óscar Chávez desde 1960, como locutor, productor de programas y radioteatro. Programas como Romance y corrido o El cine y la crítica, de Carlos Monsiváis, le permitieron a Chávez difundir su trabajo.

El contacto que estableció con Monsiváis -quien asistió como jurado del Premio Casa de las Américas en el mismo año del Encuentro, 1967-, finalmente redundó en permitirle ir al Primer Encuentro de la Canción Protesta: "Estaba él, entre mucha otra gente. Y surge el evento este que organizan los cubanos en el año 67. Y Carlos, con los nexos que él tenía, me recomienda a mí. Para ir al festival de Protesta, ahí sí de Protesta. Tons' fuimos Pepe González Márquez y yo, al festival de Cuba."

Sin embargo, a pesar de haber sido el único cantante mexicano invitado a participar en el Encuentro, la obra de rescate de la tradición que Chávez realizaba, se enmarcaba en un contexto y una tradición más amplios, como él mismo recordaba. Pues junto a González Márquez y grupos como Los Folkloristas o la cantante Judith Reyes, habían emprendido desde finales de los años cincuenta y principios de los sesenta una revaloración de la música popular mexicana. Este proceso, Chávez se lo planteaba como una revisión histórica en busca de tomar conciencia de "lo propio" desde una visión joven enmarcada en espacios culturales, artísticos y universitarios: "entonces, al empezar a cantar en ámbitos estudiantiles, comenzamos a cantar canciones políticas, históricas. De la Colonia, la Independencia, la Reforma, la Revolución... Y eso mismo me llevó, en mi caso, a empezar a opinar sobre la situación presente." ${ }^{4}$

Por otro lado, el caso de Judith Reyes es interesante por el papel que desempeñó como cantante de protesta en la vinculación con los movimientos obreros, campesinos y populares a través del corrido, que a lo largo de la historia de México ha funcionado como "instrumento de comunicación popular y de movilización ideológica” (Giménez, 1991, p. 46). Por ejemplo, durante el

\footnotetext{
3 Óscar Chávez, entrevista citada.
}

${ }^{4}$ Óscar Chávez, entrevista citada. 
siglo XIX se compusieron corridos para ensalzar caudillos, criticar presidentes o denunciar condiciones de explotación, como uno de 1904, cantado entre mineros, Minas de Esperanza, que denunciaba: "En esas minas de Esperanza / sólo Dios es español” (Giménez, 1991, p. 52).

Desde luego, el gran momento del corrido fue la revolución mexicana iniciada en 1910, cuando varios impresores de estas canciones participaron en lo que Catalina H. de Giménez (1991) ha llamado una guerrilla de corridos: "El corrido revolucionario acompañó en el plano ideológico a los hechos de armas, alimentando la moral de las tropas y defendiendo la justicia de sus reclamos" (p. 55). La tamaulipeca Judith Reyes abrevó fuertemente de esta tradición para acompañar diversas movilizaciones contemporáneas, labor a la que se comprometió con gran diligencia, tal como ella lo describía al recordar su encuentro en 1959 con un grupo de campesinos en Chihuahua, que protestaba en contra de los latifundistas y por el asesinato de uno de sus compañeros. Los miembros de la llamada "Caravana del hambre" le pidieron a Reyes: "Señorita, usté que es periodista, escriba de nuestro problema. Escriba de lo que ve aquí esta noche. ¡Mire, semos víctimas de la Reforma agraria...! [sic]”, a lo que ella respondió: “¡Sí, señor! Yo voy a escribir sobre sus problemas. Y no sólo voy a escribir... ¡lo voy a cantar! ¡Voy a escribir canciones sobre todas las cosas que veo entre ustedes! ¡Se lo prometo! Es una promesa que les hago a todos" (Reyes, 2019, p. 78).

Desde aquel momento, Judith Reyes acompañó en la lucha a una gran cantidad de movilizaciones de diversas formas, acopiando víveres, negociando con autoridades, generando espacios periodísticos para darles voz, y claro, cantando. En sus composiciones, había alusiones a luchas agrarias; denuncias antiimperialistas como la invasión que hizo Estados Unidos sobre la Bahía de Cochinos, en Cuba, en 1961; corridos revolucionarios como "Yo soy soldado de Pancho Villa", "El corrido de Cananea" o "El corrido de Arturo Gámiz" que compuso en honor a quien comandó a los jóvenes guerrilleros que participaron en el trágicamente fallido asalto al Cuartel Madera en Chihuahua en 1965. Judith Reyes tuvo tal arraigo popular que incluso fue candidata en 1964 a senadora por el Frente Electoral del Pueblo, lo cual le trajo como consecuencia diversos tipos de represión, tortura y pisar la cárcel en distintos momentos.

También a mediados de los años sesenta, Judith Reyes (2019) pudo editar su primer disco, y recuerda que "cuando tuve el primer disco en mis manos lo contemplé emocionada y exclamé: 'Éste es para Fidel'... y se lo mandé 
a Castro. No supe si lo recibió" (pp. 178-179). Aunado a las composiciones que Reyes había dedicado a Cuba a lo largo de los años sesenta, esta anécdota es elocuente de la atracción y fascinación que la revolución en la isla generaba, para una cantante de corridos políticos y canciones de protesta cercanamente vinculada a la protesta y los movimientos sociales. Lo anterior haría inexplicable que no hubiera estado en el Encuentro, de no ser por la referencia a la que tenemos acceso a través de su autobriografía, en la que narra que la noche del 17 de julio de 1967 -es decir unas semanas antes del Encuentro- la policía secuestró a su esposo por su participación en la huelga de choferes de la línea Peralvillo-Cozumel, por lo que Reyes recordaba: "Mi vida se desenvolvía en torno a la prisión de Lecumberri. Diariamente estaba allí haciendo cola para verlo" (Reyes, 2019, p. 227).

La proximidad de Judith Reyes con los movimientos sociales la colocó en una esfera de desenvolvimiento distinta a la del ámbito intelectual y universitario en la que Óscar Chávez se movía. Fue hasta 1968, con el movimiento estudiantil, que Reyes también se convirtió en una de las principales referencias de la vertiente musical de dicho proceso, igual que Chávez, Los Folkloristas, Enrique Ballesté y algunos más. La diferencia de espacios de acción durante los sesenta, habla también del espectro de vinculaciones utilizados por la Casa de las Américas para elegir a sus invitados, pues como mencionó Chávez, fue Monsiváis - un intelectual con quien compartía espacios de trabajo universitarios- quien lo vinculó para poder asistir al Primer Encuentro de la Canción Protesta.

La experiencia de Óscar Chávez fue la del joven músico talentoso que pudo entrar en contacto con sus grandes referentes artísticos por medio del Encuentro. La fascinación que le produjo esta posibilidad lo llevó a preservar la memoria histórica del evento: "la guitarra que yo llevaba, le pedí firmas a todos los que me encontrara yo en el festival. Cuando terminó el evento le dejé la guitarra a Casa de las Américas. Entonces, supongo que ahí está. Con la firma de toda la bola de cabrones". ${ }^{5}$

Dentro de su repertorio en el Primer Encuentro de la Canción Protesta, Óscar Chávez y José González Márquez presentaron piezas de la canción tradicional mexicana, como el corrido "La carabina 30-30". Pero también incorporaron composiciones musicales adaptadas de la obra poética del cubano José Martí, como "La niña de Guatemala" o "Mi honda es la de David”, piezas

${ }^{5}$ Óscar Chávez, Entrevista citada. 
que además fungen como un claro ejemplo de la paradoja entre "la rosa y la espina" de Rostgaard. Por un lado, la rosa del romanticismo trágico del desamor en "La niña de Guatemala" (Chávez, 1972):

\author{
Eran de lirios los ramos, \\ Y las orlas de reseda \\ Y de jazmín: la enterramos \\ En una caja de seda \\ Ella dio al desmemoriado \\ Una almohadilla de olor: \\ Él volvió, volvió casado: \\ Ella se murió de amor
}

Por otro lado, la espina en la consigna de inspiración bíblica cimentada en la derrota del gigante por parte de los pequeños, al remarcar el valor y potencial fortaleza de las composiciones líricas en "Mi honda es la de David" (Chávez, 1972):

$$
\begin{gathered}
\text { Mi verso es como un puñal } \\
\text { que por el puño echa flor } \\
\text { mi verso es un surtidor } \\
\text { que da un agua de coral } \\
{[\ldots . . .]} \\
\text { Mi verso al valiente agrada } \\
\text { mi verso breve y sincero } \\
\text { es del vigor del acero } \\
\text { con que se funde la espada }
\end{gathered}
$$

Además, también comenzaron sus primeras incursiones directamente en la canción de protesta, con piezas como "Historia del hombre muerto" y "Caña".

Para Chávez, el sentido del rescate de la música popular y la canción de protesta estaba en "la dignificación de nuestra música". Aunque "no esencialmente la política. Aparte desarrollábamos música romántica, de nuevos compositores. Surgía la nueva trova cubana, Silvio, Pablo, toda esta caterva" . ${ }^{6}$

${ }^{6}$ Óscar Chávez, entrevista citada. 
La dignificación de la música popular tenía como componente central la consigna antiimperialista: "Pues frente al imperio... ¡Evidentemente! Que no es uno, son una bola de cabrones. Contra las... Aunque suena panfletario, pero así es... Contra las barbaridades de los imperialistas". ${ }^{7}$

A su regreso a México, Óscar Chávez continuó conjugando sus carreras musical, radiofónica y teatral. El teatro cabaret fue el espacio en el que pudo consolidar el empalme de sus posturas políticas con sus composiciones musicales. En el teatro Blanquita grabó el primero de sus discos, Parodias políticas, en 1975. Una serie de casi una decena de álbumes en los que utilizó como base la canción popular mexicana para alterar las composiciones líricas y criticar aspectos del acontecer mexicano. Quizá la más famosa fue "La Casita", que aludía a las exorbitantes riquezas patrimoniales de los políticos mexicanos (Chávez, 1975):

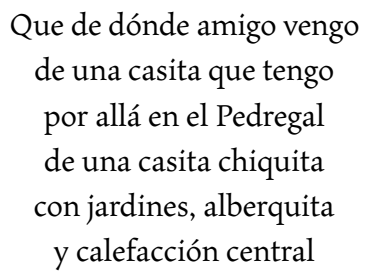

El caso de Óscar Chávez permite observar la evolución artística de un joven que asistió al Encuentro Internacional de la Canción Protesta en 1967. El tránsito a la politización tuvo como semilla el antiimperialismo cultural implícito en el rescate de la canción tradicional mexicana.

Por otro lado, el ejemplo del Encuentro o los festivales chilenos que le precedieron, fungieron también como un correlato antiimperialista de los festivales de rock de inspiración anglosajona, promovidos por los medios de comunicación masiva en algunos países latinoamericanos. Si bien se señalaron algunas conexiones previas entre los músicos -principalmente sudamericanos-, es indudable que el Encuentro fue un hito para conectar representantes de esta vertiente de la música política tanto en Cuba como en el resto de América Latina.

El Primer Encuentro Internacional de la Canción Protesta marcó el inicio de un movimiento latinoamericano de músicos y artistas antiimperia-

${ }^{7}$ Óscar Chávez, entrevista citada. 
listas que reivindicaron principios cercanos a los postulados por la "Resolución final del Encuentro de la Canción Protesta”. Es decir, la solidaridad de los creadores y artistas con diversas causas políticas. En el caso específico de América Latina, con los diversos movimientos revolucionarios que se sucedieron durante la segunda mitad del siglo xx, a partir del impulso que les dio el triunfo de la revolución cubana.

Por otro lado, la Casa de las Américas se convirtió, a partir de entonces, en un gran eje de encuentros, reuniones, conciertos y producción de fonogramas de esta corriente musical en los años setenta y ochenta con colecciones como: Palabra de esta América -enfocada en difundir la literatura latinoamericana-, la colección Nuestros Países, o la colección En Vivo en Casa, entre otros sencillos y LPs; sin dejar atrás el impulso al Movimiento de la Nueva Trova abanderado por los entonces muy jóvenes Silvio Rodríguez, Pablo Milanés y Noel Nicola; así como Leo Brouwer, Enrique Plat y Eduardo Ramos.

En México, el movimiento estudiantil de 1968 fue uno de los escenarios en los que las expresiones de la canción de protesta comenzaron a tener mayor difusión en el sentido del framing que retomamos de Paul Almeida (2019), pues "las marchas, los mítines, las asambleas, o los simples encuentros entre activistas y sociedad fueron los escenarios para una serie de manifestaciones musicales que entonces fueron conocidas como 'canciones de protesta', 'canciones sociales' o 'canciones comprometidas'” (Pérez Montfort, 2007, p. 186).

Pepe Ávila, uno de los miembros fundadores de Los Folkloristas, recuerda haber participado en algunos espectáculos en los que él interpretaba canciones de rock del grupo The Beatles, pero pronto le "pareció una incongruencia estar en el lingo-lingo, mientras todo eso pasaba fuera". Y el rock quedó en segundo plano frente a la interpelación de solidaridad. Para este grupo mexicano, el folclore adquirió una serie de implicaciones artísticas, culturales, sociales e incluso políticas. En los años sesenta "quizá una palabra redondee todo: verdad. Yo creo que ahí. Era [una música] de adentro, sin forzamientos, sin maquillajes, sin concesiones. Era lo más auténtico, directo y puro."

${ }^{8}$ Entrevista al músico y compositor José Ávila, realizada por Álvaro Vázquez Mantecón y Juan Alberto Salazar Rebolledo, 2 de agosto de 2018. Casa del Lago, Ciudad de México, México. Entrevista realizada para la exposición Vanguardia y Desacato: La Casa del Lago y el 68. 
Durante la actividad del movimiento estudiantil, Los Folkloristas presentaron un amplio repertorio de canciones del folclore latinoamericano y algunas canciones de protesta en los mítines, Óscar Chávez interpretó algunos corridos tradicionales o adaptados y Enrique Ballesté composiciones de su autoría que incluían consignas políticas antiimperialistas. Por su parte, Judith Reyes "compuso una buena cantidad de corridos que iban desde la parodia hasta la narración puntual de las tragedias suscitadas por la represión" (Pérez Montfort, 2007, p. 187).

Si bien, "ninguna canción soluciona nada", en palabras de Óscar Chávez, "la canción, como el vehículo que es, como la herramienta que es, sí es muy rica y muy útil. Puede hacer tomar conciencia." Así, la dignificación de la música popular nacional y continental, la defensa de la memoria y la búsqueda de la toma de conciencia, fueron los principales ejes rectores de la consigna antiimperialista solidaria abanderada por los jóvenes artistas reunidos en torno al Encuentro Internacional de la Canción de Protesta de 1967.

\section{LISTA DE REFERENCIAS}

Almeida, P. (2019). Social movements. The structure of collective mobilization. Oakland: University of California Press.

Ayala, R. (2013). Cosechero (CD). Buenos Aires, Argentina: Los Años Luz Discos.

Benmayor, R. (1981). La "Nueva Trova": New Cuban song. Latin American Music Review, 2(1), 11-44.

Cafrune, J. (1964). Zamba de mi esperanza [canción]. En Emoción, canto y guitarra (Vinyl). Buenos Aires, Argentina: CBs.

Carvalho-Neto, P. de (1973). El folklore de las luchas sociales. México: Siglo XXI.

Chávez, Ó. (1963). Herencia lírica mexicana (Vinyl). Ciudad de México, México: Polydor.

Chávez, Ó. (1972). Versos sencillos cantados por Óscar Chávez (Vinyl). La Habana, Cuba: Empresa de Grabaciones y Ediciones Musical.

Chávez, Ó. (1975). La casita [canción]. En Parodias políticas (Vinyl). Ciudad de México, México: Polydor.

9 Óscar Chávez, entrevista citada. 
Fairley, J. (2013). "There is no revolution without song": new song in Latin America. En B. Kutsche y B. Norton (eds.), Music and protest in 1968 (pp. 119-136). Nueva York: Cambridge University Press.

Fornaro Bordolli, M. y Malán, F. (2017). Protesta, siempre: un panorama y tres casos uruguayos. Boletín Música, 45, 41-71.

García, M. y Greco, M. (2017). “Coplera del viento”. La presencia de Óscar Matus y del Nuevo Cancionero en el Encuentro de la Canción Protesta de 1967. Boletín Música, 45, 25-39.

Gilman, C. (2012). Entre la pluma y el fusil. Debates y dilemas del escritor revolucionario en América Latina. Buenos Aires: Siglo XXI.

Giménez, C. H. de (1991). Así cantaba la revolución. México: Grijalbo.

González, J. (2017). Chile y los festivales de la canción comprometida (1955-1981). Boletín Música, 45, 5-23.

Iber, P. (2015). Neither peace nor freedom. The cultural cold war in Latin America. Boston: Harvard University Press.

Lynskey, D. (2011). 33 revoluciones por minuto. Historia de la canción protesta. Barcelona: Malpaso.

Microbiografías y opiniones de los participantes (1968). Canción Protesta, 1, 34-47.

Morejón Arnaiz, I. (2010). Política y polémica en América Latina. Las revistas Casa de las Américas y Mundo Nuevo. México: Educación y Cultura.

Nicola, N. (1975). ¿Por qué nueva trova? Caimán Barbudo, 92, 10-12.

Parra, Á. (1965). Que vivan los estudiantes [canción]. En Ángel Parra y su guitarra (Vinyl). Santiago, Chile: Demon.

Pérez Montfort, R. (2007). La música popular mexicana en 1968. En A. Vázquez Mantecón (comp.), Memorial del 68 (pp. 176-191). México: unAm/Secretaría de Cultura-GDF/Turner.

Pettinà, V. (2018). La guerra fría en América Latina. México: El Colegio de México. Reyes, J. (2019). La otra cara de la patria. Autobiografía. México: UnAM.

Rojas, R. (2018). La polis literaria. El boom, la revolución y otras polémicas de la guerra fría. México: Taurus.

Resolución final del Encuentro de la Canción Protesta (1967). Canción Protesta, 1, I-III. Siegmeister, E. (1999). Música y sociedad. México: Siglo XXI.

Spener, D. (2016). We shall not be moved/No nos moverán: Biography of a song of struggle. Filadelfia, Pensilvania: Temple University Press.

Tejeda, D. (2017). Un palimpsesto llamado Nueva Canción. Boletín Música, 45, 73-80.

Velasco, F. (2007). La Nueva Canción Latinoamericana. Notas sobre su origen y definición. Presente y Pasado. Revista de Historia, 12(23), 139-153. 
Última asamblea de la Canción Protesta (1967). Canción Protesta, 1, 30-31.

Viglietti, D. (1968). El encuentro. Canción Protesta, 1, 14-23.

Viglietti, D. (1969). Canción para mi América [canción]. En Varios Artistas, Canción protesta (Vinyl). La Habana, Cuba: Casa de las Américas.

Vilches, P. (2004). De Violeta Parra a Víctor Jara y Los Prisioneros: recuperación de la memoria colectiva e identidad cultural a través de la música comprometida. En Latin American Music Review/Revista de Música Latinoamericana, 25(2), 195-215. 